\title{
Ignition of a Reactive Solid by an Inert Hot Spot
}

\author{
A. Liñan - M. Kindelan
}

\begin{abstract}
A theoretical analysis is presented for the description of the ignition of a reactive media by inert hot bodies of finite size, when the activation energy of the reaction is large. The analysis leads to closed-form relations for the minimum "critical" size of the hot spot resulting in ignition and for the ignition time by hot spots of supercritical size. The analysis is carried out, first, for inert spots with heat conductivities and diffusivities of the order of those of the reactive media, and, second, for inert bodies of large conductivity.
\end{abstract}

\section{Introduction}

\begin{abstract}
A SYMPTOTIC techniques have been very useful to describe ignition under different heating mechanisms. The objective of this work is to apply these techniques to the case in which a cylindrical or spherical spot of inert material embedded in an infinite reactive material is heated suddenly at time zero to a temperature $T_{i}$ higher than the temperature $T_{0}$ of the surrounding reactive material.

This ignition model is of interest in connection with the problem of hot-wire ignition and with the problem of ignition of explosives by gap compression. Hot-wire ignition theories have been proposed by Friedman (1969) and by Baer and Ryan (1970). These authors consider the case in which the wire is heated by a current of constant energy and integrate the energy conservation equation, either by approximate techniques or by numerical methods. Our asymptotic analysis models the case in which a certain electric energy is circulated through the wire in a time short compared with the characteristic thermal response time of the reactive solid. In this way the wire is heated to a high temperature $T_{i}$, prior to the occurrence of any thermal change in the solid. However, in times of the order of the characteristic thermal response time, the wire will heat up the reactive material by conduction while the thermal energy contained in the solid will decrease. In the problem analyzed by Friedman and by Baer and Ryan there is a constant energy current so that the energy supplied is unlimited and, therefore, ignition always occurs. In our
\end{abstract}


case, however, there is a finite amount of energy supplied to the wire and, therefore, ignition will occur or not occur depending on the thermal properties of the materials, amount of energy supplied, wire diameter, and chemical parameters of the condensed phase reaction.

A numerical analysis of this problem has been carried out by Goldshleger et al. (1973). An asymptotic analysis for large activation energies was carried out by Berman and Ryazantsev (1976) for the case when the conductivity of the inert body is large enough, so that its temperature can be considered a function of time only, and the density ratio is of order unity, so that when ignition occurs under supercritical conditions only a thin layer has been heated in the reactive material. We consider more general cases including the case when the ratio of thermal diffusivities of the inert and reactive material is not large; our analysis leads to closed-form relations giving the critical hot-spot size, or critical ignition energy, in terms of the physicochemical parameters of the materials.

Gap compression has been proposed by Randolph and Simpson (1973) and Randolph (1975), for example, as a mechanism responsible for the accidental ignition of high explosive charges. Accidental ignition occurs, for instance, when an explosive-filled shell explodes prematurely when accelerated during firing or when an encased high explosive charge is subjected to relatively small impacts, e.g., when dropped a few feet. Presumably, in both of these examples ignition was produced by compression heating of gas in voids or cracks due to defective explosive loading. Randolph (1975) considers different pressure rise curves and integrates numerically the equations describing the process. The problem considered in the present work models ignition by gas compression with a step-like pressure rise occurring in a time short compared with the characteristic thermal response time in the reactive solid.

\section{Formulation}

We consider a cylindrical or spherical inert spot of radius $a$ whose temperature $T$ is instantaneously raised at time zero to a value $T_{i}$ higher than the value $T_{0}$ of the reactive material. A distributed exothermic reaction of the Arrhenius type takes place in the reactive solid. The energy conservation equation can be written as

$$
\frac{\partial T}{\partial t}=\alpha\left(\frac{\partial^{2} T}{\partial r^{2}}+\frac{n}{r} \frac{\partial T}{\partial r}\right)+A \exp \left(-\frac{E}{R T}\right)
$$

assuming constant values for the density $\rho$, the specific heat $c$, and heat conductivity $k$, although not necessarily equal for the inert material, where we shall use the subscript $i$, and for the reactive medium, subscript 0 .

In Eq. (1), $n=1$ for cylindrical spots and $n=2$ for spherical spots. $E$ is the activation energy, and $A$ the pre-exponential factor of the reaction; $A=0$ in the inert material $r<a$. At $r=a$ there is a jump in the thermal diffusivity $\alpha$ from its value $\alpha_{i}=k_{i} / \rho_{i} c_{i}$ in the inert material to the value $\alpha_{0}=k_{0} / \rho_{0} c_{0}$ in the reactive medium. 
Equation (1) must be solved with the initial condition $T=T_{i}$ for $r<a$, and $T=T_{0}$ for $r>a$. The temperature and the heat flux $k \partial T / \partial r$ must be continuous at $r=a$.

When Eq. (1) is written in nondimensional form by measuring, for example, the distance $r$ with $a$, the time $t$ with $a^{2} / \alpha_{0}$, the temperature $T$ with $T_{0}$, the following parameters are found to describe the solution: a nondimensional activitation energy $E / R T_{0}$, a Damköhler number $A a^{2} / \alpha_{0} T_{0}$, the temperature ratio $T_{i} / T_{0}$, and the ratios $k_{i} / k_{0}$ and $\alpha_{i} / \alpha_{0}$ of heat conductivities and thermal diffusivities.

For large values of the nondimensional activitation energy $E / R T_{0}$, when the increment in hot-spot temperature $T_{i}-T_{0}$ is of the order of $T_{0}$, the ignition time becomes very small compared with the adiabatic ignition time $\left(R T_{0}{ }^{2} / E A\right) \exp \left(E / R T_{0}\right)$ of the reactive material, if the Damköhler number $A a^{2} / \alpha_{0} T_{0}$ is larger than a critical value that we shall try to calculate in the following in terms of $E / R T_{0}, T_{i} / T_{0}, k_{i} / k_{0}$, and $\alpha_{i} / \alpha_{0}$. This critical value has been calculated by Berman and Ryazantsev (1976) for large values of $k_{i} / k_{0}$ and values of $k_{i} \alpha_{0} / k_{0} \alpha_{i}$ of order unity. We shall consider first the case where the ratio $k_{i} / k_{o}$ is also of order unity, and then in Sec. V we shall deal briefly with the cases where $k_{i} / k_{0}$ is large, including the case when $k_{i} \alpha_{0} / k_{0} \alpha_{i}=$ $\rho_{i} c_{i} / \rho_{0} c_{0}$ is also large, when the analysis of Berman and Ryazantsev cannot be used.

We begin by an analysis of the inert solution because for large values of $E / R T_{0}$ a small increment in temperature, of the order of $R T_{0}^{2} / E$, above the temperature given by the inert solution is sufficient to increase the reaction rate by a large factor, and thus may result in ignition due to a thermal runaway. That is, the inert solution gives, for large activation energies, the temperature distribution in first approximation; whether and when ignition occurs depends on the character of the solution of the nonlinear equation describing the small perturbations to the inert solution due to the effects of the chemical reaction.

\section{Inert Solution}

The solution of the inert problem can be obtained analytically, see for instance Carslaw and Jaeger (1959), but if $n=1$, it is difficult to use since it involves complicated integrals of Bessel functions. We shall need only some details of the solution for small times, and these can be obtained more simply with the aid of asymptotic techniques.

At time zero the temperature at the surface of the spot $r=a$ rises instantaneously to an intermediate "jump" temperature $T_{j}$

$$
T_{j}=\left(T_{0}+\Gamma T_{i}\right) /(1+\Gamma)
$$

where $\Gamma=\sqrt{(k \rho c)_{i}(k \rho c)_{0}}$ is the ratio of thermal responsivities. This jump temperature is obtained at time zero at the interface of two semi-infinite slabs with different initial temperatures. In the planar case the interface temperature remains constant while the width of the heat-up zones increases with the square root of time. In the cylindrical or spherical cases, however, the in- 
terface temperature will decrease with time if $T_{0}<T_{i}$; initially, because energy from a decreasing volume is used to heat an increasing volume, and later, when the thermal wave reaches the center of the spot because of the finite amount of energy contained in the spot.

In fact, the asymptotic procedure described by Letcher (1969) can be used to calculate the temperature distribution for small times, such that $\sqrt{\alpha t}$ is much smaller than the inert spot dimension $a$; the solution can be written as expansion in powers of $\sqrt{\alpha_{0} t} / a$ times functions of the similarity variable $(r-a) / \sqrt{a_{0} t}$. Figure 1 compares this solution with the exact solution given by Crank (1956) for a spherical hot spot in an homogeneous material.

It is observed that for times small compared with the characteristic diffusion time $a^{2} / \alpha$, the quasisimilar solution follows closely the exact solution, and in particular, the approximate solution remains valid close to the surface even when the thermal wave has already reached the center of the spot.

The expansion of the inert solution for small times, near the surface is, for $r \geq a$,

$$
\frac{T-T_{j}}{T_{i}-T_{j}}=-\Gamma \frac{r-a}{\sqrt{\pi \alpha_{0} t}}-\Gamma \frac{n}{a(l+\Gamma)}\left(\sqrt{\alpha_{i}}+\sqrt{\alpha_{0}}\right) \sqrt{t / \pi}
$$

Thus, curvature effects cause the surface temperature to decrease from its jump value with the square root of time. However, the exothermicity of the chemical reaction tends to increase the surface temperature, and therefore to further accelerate the reaction rate. Ignition will or will not occur depending

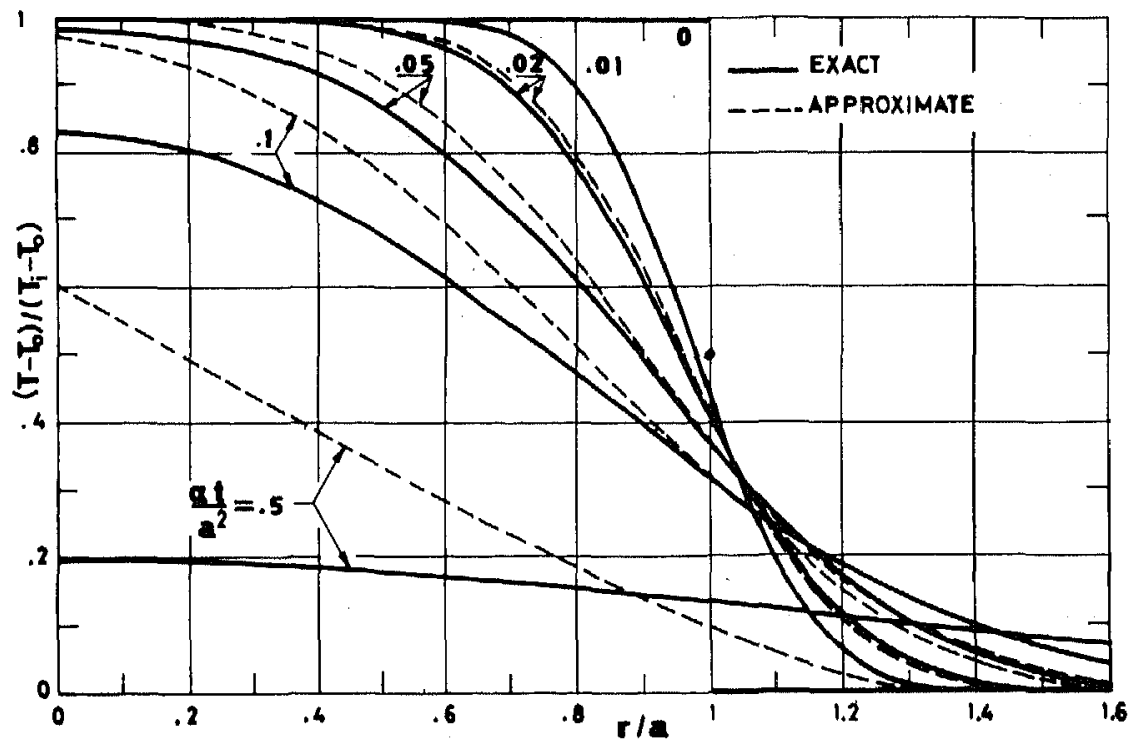

Fig. 1 Inert temperature distribution, 
on whether cooling associated with curvature effects or heating associated with the exothermicity of the chemical reaction dominates.

In the following section we shall see how the effects of chemical reaction lead to ignition for supercritical hot spots of inert materials with moderate values of the heat conductivity, such that $\Gamma$ is of order unity and $T_{j}$ is not too close to either $T_{0}$ or $T_{J}$. The case $\Gamma>1$ will be dealt with in Sec. V.

\section{Ignition Analysis for $\Gamma \sim 1$}

In the limit of high activitation energy of the Arrhenius reaction, small changes in temperature of order $R T_{j}{ }^{2} / E$ produce changes of order unity in the reaction rate. Thus, the chemical reaction term is only important in a thin region near the surface where the temperature differs from the jump temperature by a small quantity of order $R T_{j}{ }^{2} / E$. Outside of this inner zone the reaction is frozen. Figure $2 a$ is a schematic representation of this problem, showing the width of the reaction and transport layers. To describe the ignition process it is convenient to introduce as dependent variable, the deviation of the nondimensional temperature from its inert value $\theta_{I}$

$$
\chi=\beta\left(\theta-\theta_{1}\right)
$$

where

$$
\beta=E\left(T_{i}-T_{j}\right) / R T_{j}^{2}
$$

and

$$
\theta=\left(T-T_{j}\right) /\left(T_{i}-T_{j}\right)
$$

so that $\chi$ is of order unity in the reaction zone.

The characteristic time of the problem $t_{c}$ is determined from Eq. (3), by requiring that the change of temperature during time $t_{c}$ be equal to $1 / \beta$, so that

$$
t_{c}=\frac{\pi}{n^{2} \beta^{2}} \frac{a^{2}(1+\Gamma)^{2}}{\Gamma^{2}\left(\sqrt{\alpha_{i}}+\sqrt{\alpha_{0}}\right)^{2}}
$$

and therefore the characteristic variables of the process are

$$
\sigma=t / t_{c}, \quad \xi=(r-a) / \sqrt{\alpha_{0} t_{c}}
$$

In terms of these variables, Eq. (1) becomes, up to terms of order $1 / \beta$,

$$
\frac{\partial \chi}{\partial \sigma}=n \frac{\alpha}{\alpha_{0}} \frac{\sqrt{t_{c} \alpha_{\theta}}}{a} \frac{\partial \chi}{\partial \xi}+\frac{\alpha}{\alpha_{0}} \frac{\partial^{2} \chi}{\partial \xi^{2}}+\beta \Delta \exp \left(\chi-\sqrt{\sigma}-\frac{\beta \Gamma \xi}{\sqrt{\pi \sigma}}\right)
$$




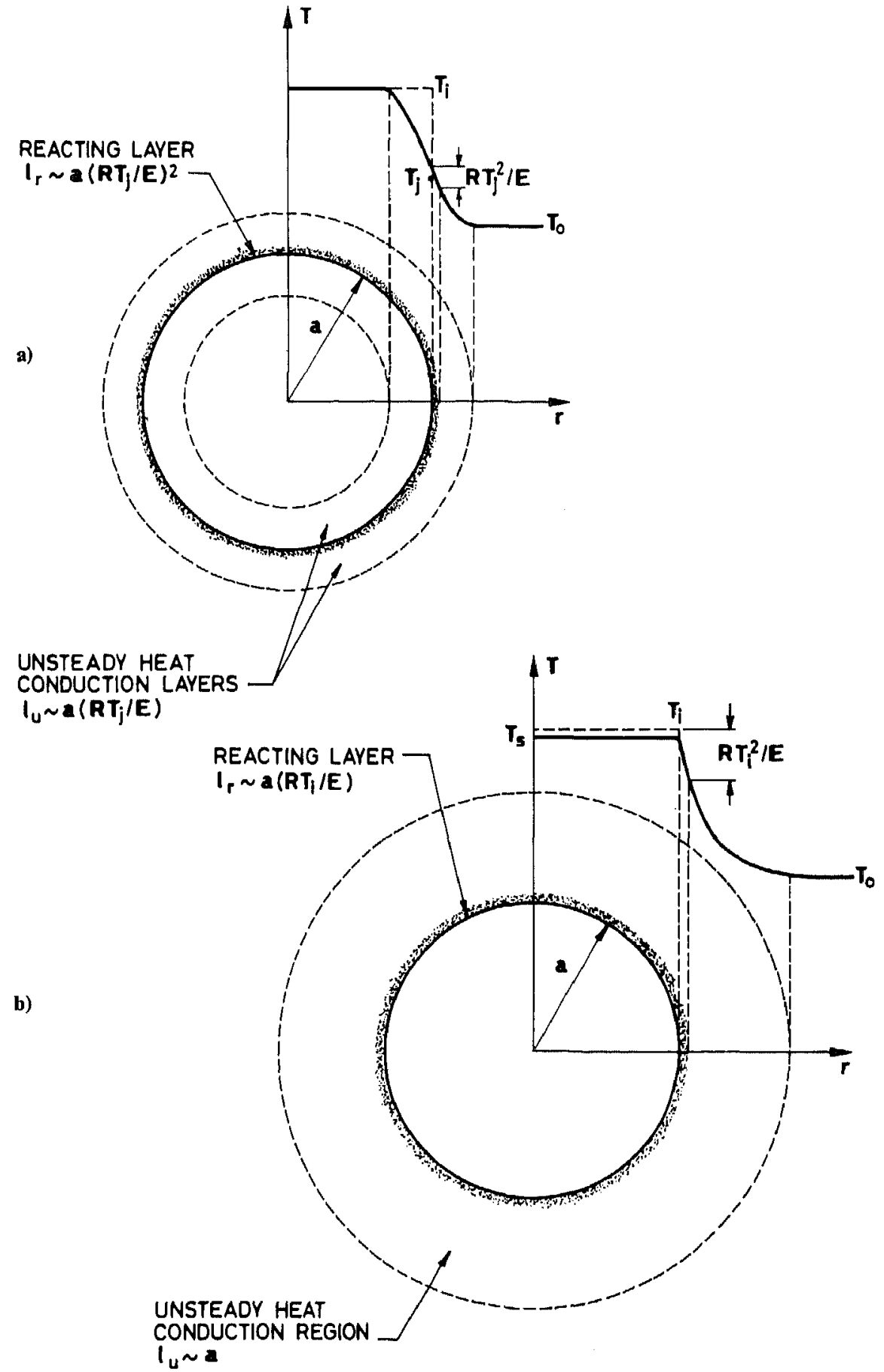

Fig. 2 Schematic representation of the problem; a) $\Gamma$ of order unity, b) $\Gamma>1$. 
where $\alpha=\alpha_{0}$ and $\Delta$, assumed of order unity, is given by

$$
\Delta=A t_{c}\left(T_{i}-T_{j}\right)^{-1} \exp \left(-E / R T_{j}\right)
$$

in the reactive zone $\xi>0$, and $\alpha=\alpha_{i}, \Delta=0$ in the inert spot $\xi<0$.

The factor $n / r$ in the first term of the right-hand side of Eq. (1) has been replaced by $n / a$, because during the ignition transient the thickness of the transport zone, of order $\sqrt{\alpha t_{c}} \sim a / \beta$, is small compared with $a$; the resulting first term in the right-hand side of Eq. (9) resembles a term due to a convective flow toward the hot spot, and its effects in the ignition process are similar to those of convection due to gasification analyzed by Kindelán and Liñán (1978). Equation (9) has to be solved with boundary conditions implying continuity of temperature and heat flux at $\xi=0$.

It should be observed that in the reactive solid $\xi>0$, there is an outer zone, where $\xi$ is of order unity, in which the chemical reaction is frozen, and an inner zone, where $\xi$ is of order $1 / \beta$, in which the effect of the chemical reaction has to be retained.

In the outer unsteady transport zone the term due to geometrical effects in Eq. (9), of order $1 / \beta$, can also be neglected in first approximation compared with the transient and heat conduction terms. Therefore, the lowest order in an asymptotic expansion in powers of $1 / \beta$ for the outer zone $\chi_{01}$ satisfies the onedimensional diffusion equation; thus its solution, for $\xi>0$, can be written in the form

$$
\chi_{0 l}=\left.\int_{0}^{0} \frac{\exp \left[-\xi^{2} / 4\left(\sigma-\sigma^{\prime}\right)\right]}{\sqrt{\pi} \sqrt{\sigma-\sigma^{\prime}}} \frac{\partial \chi_{0 I}}{\partial \xi}\right|_{s} \mathrm{~d} \sigma^{\prime}
$$

in terms of the apparent surface heat flux $\partial \chi_{01} /\left.\partial \xi\right|_{s}$, a function of $\sigma^{\prime}$, coming from the thin reaction zone.

In the inner reactive zone, the characteristic spatial variable is $y=\beta \xi$; so that $y \sim 1$ insures that the change of temperature from its surface value is, as the increment due to chemical reaction, of order 1 in $\chi$, and therefore the reaction term has to be retained.

Introducing the expansion $\chi=\chi_{1}(y, \sigma)+\beta^{-1} \chi_{2}(y, \sigma)+\ldots$ in Eq. (9), previously written in terms of the variable $y$, and collecting like powers of $\beta$, the following equations are obtained

$$
\begin{gathered}
\frac{\partial^{2} \chi_{1}}{\partial y^{2}}=0 \\
\frac{\partial^{2} \chi_{2}}{\partial y^{2}}=-\Delta \exp \left(\chi_{1}-\sqrt{\sigma}-y \Gamma / \sqrt{\pi \sigma}\right)
\end{gathered}
$$

that can be integrated to yield

$$
\frac{\partial \chi_{l}}{\partial y}=F(\sigma)
$$


and

$$
\frac{\partial \chi_{2}}{\partial y}=\left.\frac{\partial \chi_{2}}{\partial y}\right|_{0^{+}}+\frac{\sqrt{\pi \sigma}}{\Gamma} \Delta\left\{\exp \left(\chi_{1}-\sqrt{\sigma}-y \Gamma / \sqrt{\pi \sigma}\right)-\exp \left(\chi_{1}-\sqrt{\sigma}\right\}\right.
$$

if we anticipate that $F=0$.

In the inert material $\xi<0$, the solution is analogous to that of the outer zone of the reactive solid $\xi>0$, and therefore the lowest order solution, $\chi_{11}$, can be written as

$$
\chi_{i l}=\left.\sqrt{\frac{\alpha_{i}}{\alpha_{0}}} \int_{0}^{\sigma} \frac{\exp \left(-\xi^{2} \alpha_{0} / 4 \alpha_{i}\left(\sigma-\sigma^{\prime}\right)\right)}{\sqrt{\pi} \sqrt{\sigma-\sigma^{\prime}}} \frac{\partial \chi_{i l}}{\partial \xi}\right|_{0^{-}} \mathrm{d} \sigma^{\prime}
$$

in terms of the interface gradient $\partial \chi_{11} /\left.\partial \xi\right|_{0^{2}}$ evaluated at $\sigma=\sigma^{\prime}$.

The solution for the inner zone of the reactive material has to be matched on one side $y=0$ to the solution given by Eq. (16) for the inert spot, and on the other side $y \rightarrow \infty$ to the solution given by Eq. (14) for the outer unsteady transport zone. Continuity of temperature and heat flux at $\xi=0$ provide the following relationships to be used in matching with the inert spot

$$
\begin{array}{cc}
\left.\frac{\partial \chi_{I}}{\partial y}\right|_{0^{+}}=0, & \left.k_{i} \frac{\partial \chi_{i l}}{\partial \xi}\right|_{0^{-}}=\left.k_{0} \frac{\partial \chi_{2}}{\partial y}\right|_{0^{+}} \\
\chi_{i 1}(0, \sigma)=\chi_{l}(0, \sigma), & \chi_{i 2}(0, \sigma)=\chi_{2}(0, \sigma)
\end{array}
$$

From Eqs. (14) and (17) we obtain, as anticipated above,

$$
\partial \chi_{l} / \partial y=0, \quad \chi_{l}(y, \sigma)=\chi_{s}(\sigma)
$$

The matching conditions between the solutions for the inner and outer zones require

$$
\chi_{0 I}(0, \sigma)=\chi_{s},\left.\quad \frac{\partial \chi_{0 I}}{\partial \xi}\right|_{s}=\left.\frac{\partial \chi_{2}}{\partial y}\right|_{\infty}
$$

and from Eq. (15)

$$
\left.\frac{\partial \chi_{2}}{\partial y}\right|_{\infty}=\left.\frac{\partial \chi_{2}}{\partial y}\right|_{0^{+}}-\frac{\Delta}{\Gamma} \sqrt{\pi \sigma} \exp \left(\chi_{s}-\sqrt{\sigma}\right)
$$

Using Eq. (11) for $\xi=0$ we obtain

$$
\chi_{s}=-\int_{0}^{\sigma} \frac{\partial \chi_{2} /\left.\partial y\right|_{0^{+}}}{\sqrt{\pi} \sqrt{\sigma-\sigma^{\prime}}} \mathrm{d} \sigma^{\prime}+\frac{\Delta}{\Gamma} \int_{0}^{\sigma} \sqrt{\frac{\sigma^{\prime}}{\sigma-\sigma^{\prime}}} \exp \left(\chi_{s}-\sqrt{\sigma^{\prime}}\right) \mathrm{d} \sigma^{\prime}
$$


Also, from Eqs. (16) and (17),

$$
\chi_{s}=\frac{1}{\Gamma} \int_{0}^{\sigma} \frac{\partial \chi_{2} /\left.\partial y\right|_{0^{+}}}{\sqrt{\pi} \sqrt{\sigma-\sigma^{\prime}}} \mathrm{d} \sigma^{\prime}
$$

We can combine Eqs. (22) and (23) to generate the following nonlinear integral equation

$$
\chi_{s}(\sigma)=P \int_{0}^{\sigma} \sqrt{\frac{\sigma^{\prime}}{\sigma-\sigma^{\prime}}} \exp \left[\chi_{s}\left(\sigma^{\prime}\right)-\sqrt{\sigma^{\prime}}\right] \mathrm{d} \sigma^{\prime}
$$

to describe the evolution with time of the interface temperature. The parameter $P=\Delta / \Gamma(\Gamma+1)$, of order unity in the regime of transition from subcritical to supercritical conditions,

$$
P=\frac{a^{2}}{\left(\sqrt{\alpha_{o}}+\sqrt{\alpha_{i}}\right)^{2}} \frac{\pi(1+\Gamma)^{2}}{n^{2} \Gamma^{3}} \frac{A \exp \left(-E / R T_{j}\right)}{\beta^{2}\left(T_{i}-T_{j}\right)}
$$

is a measure of the cooling effect associated with the curvature of the inert hotspot.

In order to integrate numerically Eq. (24), it is convenient to introduce $s=$ $\sqrt{\sigma P}$ as the new time variable. After integrating by parts to remove the singularity at $\sigma^{\prime}=\sigma$, the equation is written in a finite-definite form, so that at each new step an implicit algebraic equation in the unknown $\chi_{s}(s)$ is solved by a Newton-Raphson procedure. The resulting increment in interface temperature $\chi_{s}$ due to the chemical reaction is shown in Fig. 3 as a function of the modified time variable $s$ for different values of $P$. A critical value $P_{\mathrm{c}}=0.381$ of the parameter $P$ is found such that for values of $P$ smaller than $P_{c}$ the surface temperature increment $\chi_{s}$ goes through a maximum at $s=s_{\max }(P)$ and ignition does not take place; for values of $P$ larger than $P_{c}$, a runaway of the interface temperature occurs at a finite ignition time, $s_{\mathrm{ign}}$, which is shown together with $s_{\max }$ as a function of $P$ in Fig. 4. For values of $P<P_{c}$ the cooling of the interface due to geometrical effects dominates over the heating due to the exothermic chemical reaction, while for $P>P_{c}$ the last effect dominates. It is observed that when $P$ approaches $P_{c}$, both the ignition time and the time when the temperature increment $\chi_{s}$ is maximum tend to infinity.

Notice that $P \rightarrow \infty$ in the planar case, so that our results cannot be used to calculate the critical hot-spot size if it is in the form of a slab; in this case, analyzed numerically by Vilyunov and Kolchin (1966), the critical conditions are given in order of magnitude by $a=\sqrt{\alpha_{i} t_{c}}$.

\section{Critical Conditions for $\Gamma \gg 1$}

The analysis of the previous section was carried out under the assumption that $k_{i} / k_{0}$ and $(\rho c)_{i} /(\rho c)_{0}$, and therefore $\Gamma$, are of order unity. If the ratio $k_{i} / k_{0}$ is large, $\Gamma$ becomes large and the previous results are not applicable, when ignition takes place at times $t_{c}$ such that $\sqrt{\alpha_{i} t_{c}} \geqslant a$. Berman and 


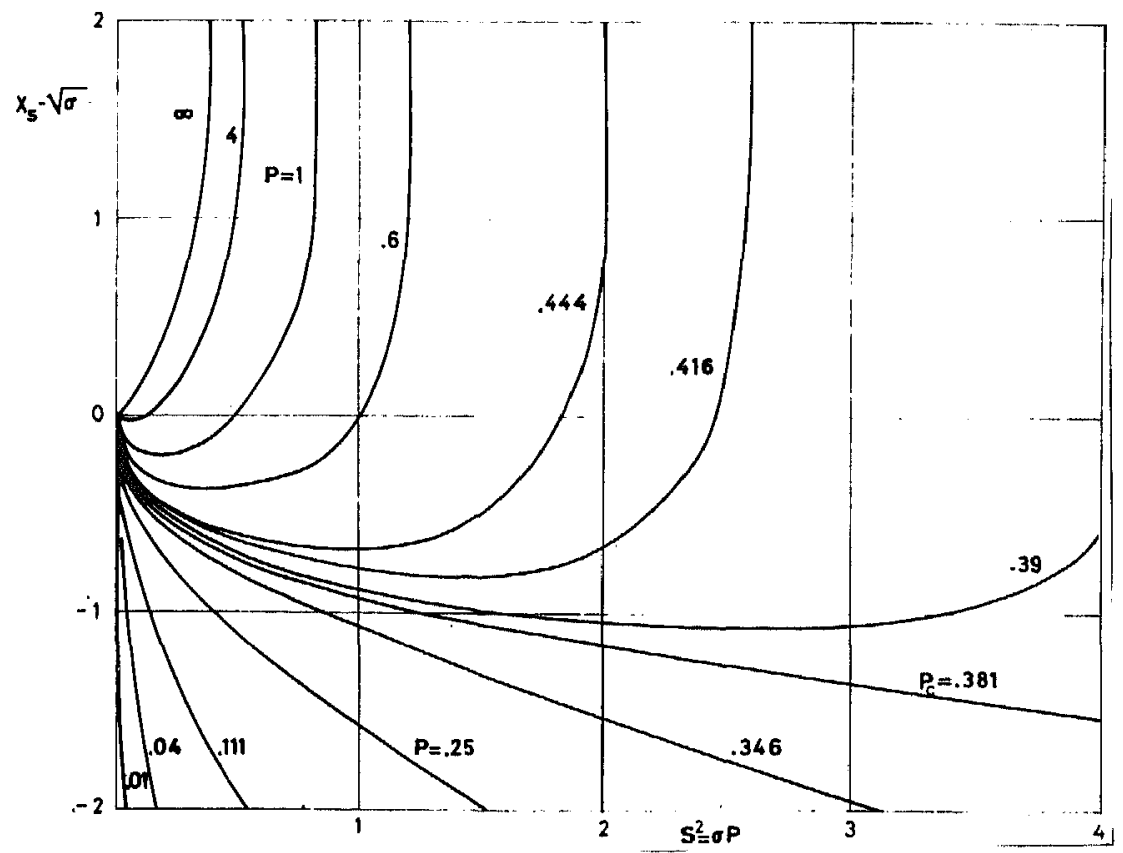

Fig. 3 Non dimensional interface temperature as a function of the non dimensioinal time $s^{2}=\sigma P$ for different values of the geometrical parameter $P$.

Ryazantsev (1976) used asymptotic techniques to calculate, for large activation energies, the critical hot-spot size in the limiting case $k_{l} / k_{0} \rightarrow \infty$ and $(\rho c)_{i} /(\rho c)_{0}$ of order unity. We generalize their analysis here to include the cases where $(\rho c)_{i} /(\rho c)_{0}$ is large, assuming as they did that $\sqrt{\alpha_{i} t_{c}}>a$.

In the limit $k_{l} / k_{0} \rightarrow \infty$, the temperature can be considered to be uniform $T=T_{s}(t)$ in the inert spot during most of the ignition transient, so that Eq. (1) must be solved for $r>a$ with the boundary conditions at $r=a$ replaced by

$$
r=a: \quad T=T_{s}, \quad \frac{\partial T}{\partial r}=\frac{\rho_{i} c_{i} a}{(n+I) k_{0}} \frac{\mathrm{d} T_{s}}{\mathrm{~d} t}
$$

If this problem is written in terms of the nondimensional variables $x=r / a$, $\tau=\alpha_{0} t / a^{2}$, and $T / T_{0}$ mentioned in the Introduction, the parameters $k_{i} / k_{0}$ and $\alpha_{i} / \alpha_{0}$ appear in the solution only through the combination $(\rho c)_{i} /(\rho c)_{0}$. We shall look for the asymptotic description, for large values of $\epsilon^{-1}=E / R T_{i}$, of the ignition process in near critical conditions in the distinguished limiting case where

$$
\lambda=\frac{T_{i}}{T_{i}-T_{0}} \frac{R T_{i}}{(n+1) E} \frac{(\rho c)_{i}}{(\rho c)_{0}}
$$




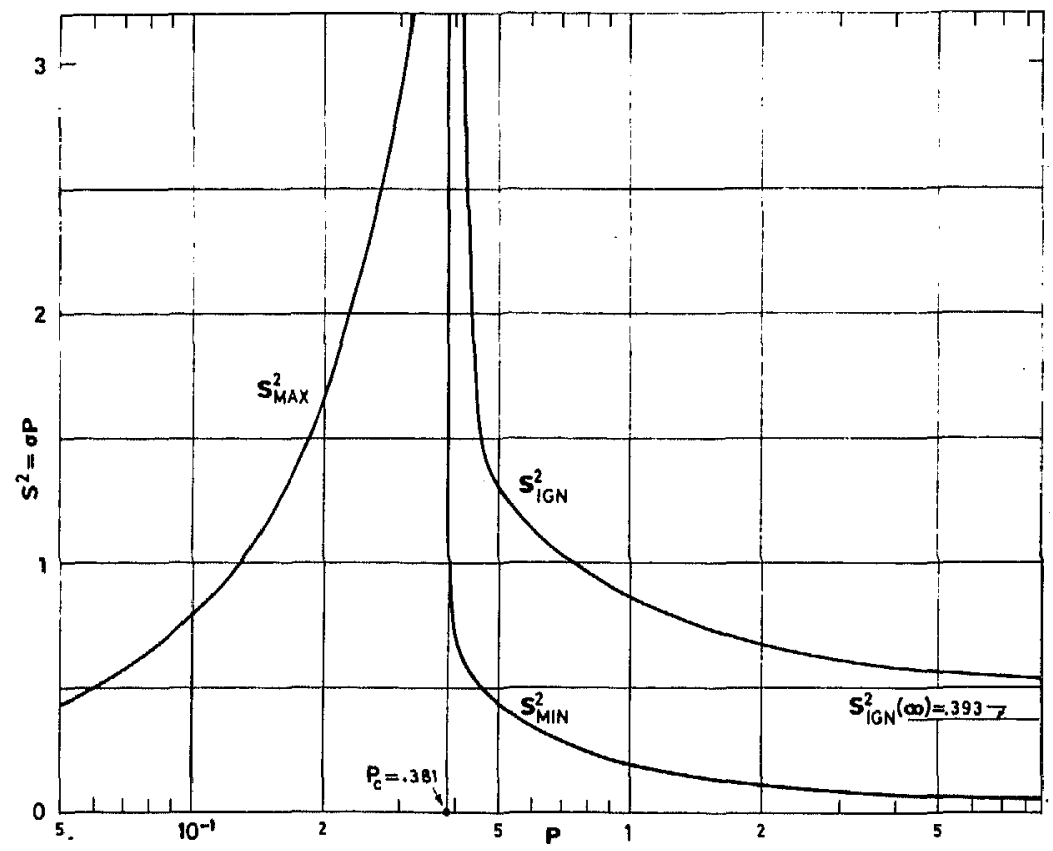

Fig. 4 Nondimensional ignition time $s_{\text {ign }}^{2}$ and time of maximum and minimum interface temperature $s_{\max }^{2}$ and $s_{\min }^{2}$.

is of order unity. In this case ignition under supercritical conditions occurs at a time of order $a^{2} / \alpha_{0}$, when the thermal heating of the reactive material has reached distances of order $a$; the chemical reaction is still confined to a thin layer of thickness of the order $a \epsilon$, adjacent to the interface with the hot spot, where $T-T_{i} \sim \epsilon\left(T_{i}-T_{0}\right)$. Figure $2 \mathrm{~b}$ shows a schematic representation of this problem.

When the temperature in the outer frozen region is expanded in powers of $\epsilon$, we obtain the following expression

$$
\left.\left(T-T_{0}\right) / T_{i}-T_{0}\right)=\Phi(x, \tau)
$$

if terms of order $\epsilon$ are neglected in the right-hand side of Eq. (28). This is the temperature distribution in the reactive material for $A=0$ and with the temperature of the hot spot kept constant at the value $T=T_{i}$ for $t>0$. The solution to this problem is described by Crank (1956) for the spherical case. From the solution we need to calculate

$$
\left.\frac{a}{\left(T_{i}-T_{0}\right)} \frac{\partial T}{\partial r}\right|_{r=a}=\left.\frac{\partial \Phi(x, \tau)}{\partial x}\right|_{x=1}=-g(\tau)
$$

which in the spherical case is given by $g(\tau)=1+1 / \sqrt{\pi \tau}$. 
For the thin reaction zone we use as independent variables $\tau$ and $\eta=$ $(r-a) / \epsilon a$. For $T$ we assume the expansion

$$
T=T_{i}(1+\epsilon \phi(\eta, \tau)+\ldots)
$$

and obtain the following differential equation for $\phi$

$$
\frac{\partial^{2} \phi}{\partial \eta^{2}}+\frac{\left(T_{i}-T_{0}\right)^{2}}{2 T_{i}^{2}} \delta e^{\phi}=0
$$

representing a balance between the chemical reaction and heat conduction terms, if

$$
\delta=2 \frac{R T_{i}}{E}\left(\frac{T_{i}}{T_{i}-T_{0}}\right)^{2} \frac{A a^{2}}{\alpha_{0} T_{i}} \exp \left(-E / R T_{i}\right)
$$

The boundary conditions at the interface $\eta=0$ with the hot spot lead to

$$
\eta=0: \quad \phi=\phi_{s}(\tau), \quad \frac{\partial \phi}{\partial \eta}=\lambda \frac{T_{i}-T_{0}}{T_{i}} \frac{\mathrm{d} \phi_{s}}{\mathrm{~d} \tau}
$$

The matching conditions with the solution for the outer frozen region provide the condition

$$
\left.\frac{\partial \phi}{\partial \eta}\right|_{\eta \rightarrow \infty}=-\left(1-T_{0} / T_{i}\right) g(r)
$$

These two conditions together with Eq. (31) lead to the differential equation

$$
\lambda \frac{\mathrm{d} \phi_{s}}{\mathrm{~d} \tau}=-\left\{g^{2}(\tau)-\delta e^{\phi_{s}}\right\}^{\not h}
$$

When this equation is integrated with the condition $\phi_{s}(0)=0$ for a fixed value of $\lambda$ and various $\delta$, we find that $\phi_{s}$ is a decreasing function of $\tau$ for all times if $\delta<\delta_{c}(\lambda)$, while the right-hand side of Eq. (35) becomes imaginary if $\delta>\delta_{c}$ after a time $\tau_{I}(\lambda)$ that can be considered as the ignition time.

The analysis of Berman and Ryazantsev (1976) corresponds to the case $\lambda \ll 1$, for which ignition occurs at small $\tau$, when $g(\tau)=1 / \sqrt{\pi \tau}$ and $\phi_{s}$ is given by the solution of the limiting form

$$
\lambda \frac{\mathrm{d} \phi_{s}}{\mathrm{~d} \tau}=-\left(1 / \pi \tau-\delta \exp \phi_{s}\right\}^{1 / 2}
$$

of Eq. (35) for small $\lambda$. If $\delta$ is larger than $\delta_{c}=0.312 \lambda^{-2}$, the solution shows a thermal runaway occurring at an ignition time $\tau_{I} \lambda^{2}$ which is a function of $\delta \lambda^{2}$. 


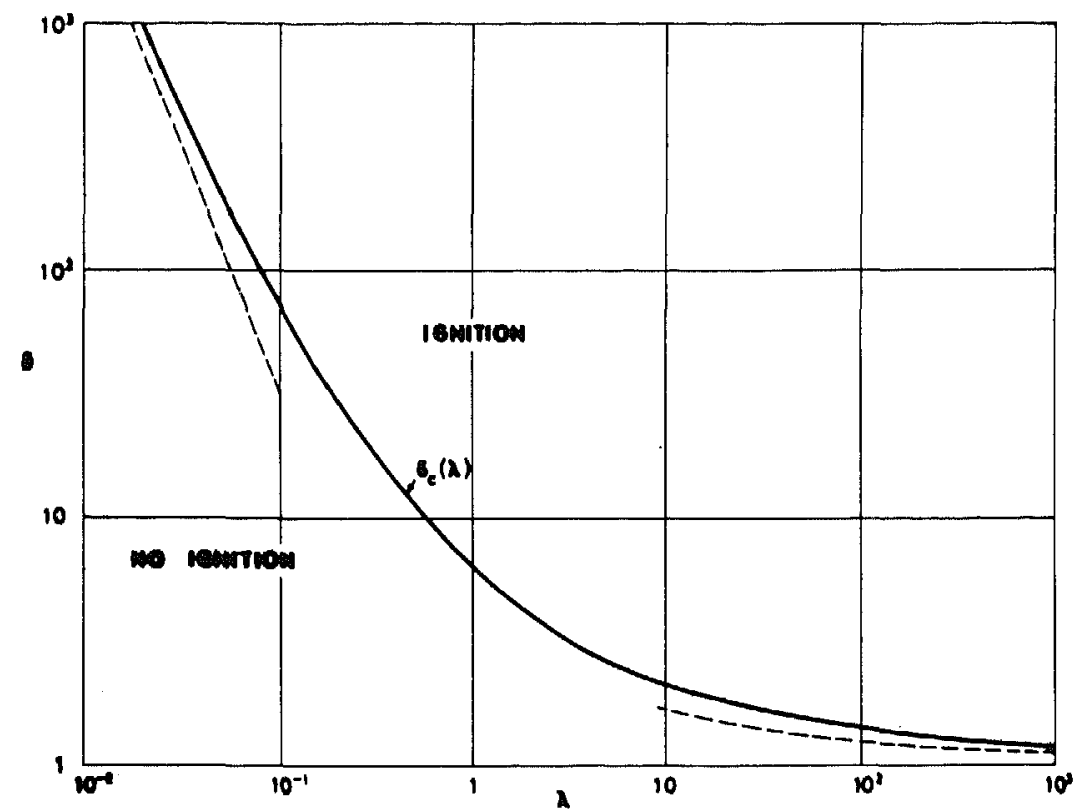

Fig. 5 Critical value $\delta_{c}(\lambda)$ for ignition by hot wires when $\Gamma>1$. Asymptotic expressions $\delta_{c}=0.31 .2 \lambda^{-2}$ for $\lambda<1$ and $\delta_{c}=1+1.8 \lambda^{-2 / 5}$ for $\lambda \geqslant 1$.

In the opposite limiting case $\lambda \gg 1$, it is easy to show that $\delta_{c} \rightarrow 1$ in the spherical case, when $\phi_{s}$ is given by

$$
\lambda \phi_{s}=-\int_{0}^{\pi}\left\{(1+1 / \sqrt{\pi \sigma})^{2}-\delta\right\}^{1 / 2} \mathrm{~d} \sigma
$$

Figure 5 shows $\delta_{c}$ for spherical spots as a function of $\lambda$, as well as the asymptotic limits for $\lambda$ large and small.

\section{Conclusions}

We have carried out an asymptotic analysis, for large activitation energy of the problem of ignition of reactive materials by hot inert bodies of finite size with the aim of obtaining closed-form expressions for the ignition time and for the critical body size resulting in ignition. This problem is a classical problem in the "Theory of Thermal Ignition" reviewed by Merzhanov and Averson (1971).

In Sec. IV we considered the case where the ratios of heat conductivities $k_{i} / k_{0}$ and heat capacities $\rho_{i} c_{i} / \rho_{0} c_{0}$ are both of order unity. A nonlinear integral Eq. (24) was found for the description of the interface temperature, given in terms of $\chi_{s}$ by

$$
\frac{T_{s}-T_{j}}{T_{i}-T_{j}}=-\frac{n \Gamma}{I+\Gamma} \cdot \frac{\sqrt{\alpha_{i}}+\sqrt{\alpha_{0}}}{a} \sqrt{\frac{\tau}{\pi}}+\frac{R T_{j}^{2}}{E\left(T_{i}-T_{j}\right)} \chi(s)
$$


with

$$
s^{2}=\frac{t A}{\left(T_{i}-T_{j}\right)} \frac{\exp \left(-E / R T_{j}\right)}{\Gamma(\Gamma+1)}
$$

The function $\chi_{s}$ of time $s^{2}$ and $P$, defined in Eq. (25), is represented in Fig. 3. The parameter $P$ measures the cooling effects due to the finite body size. For supercritical hot spots corresponding to values of $P>P_{c}=0.381$ a thermal runaway occurs at an ignition time $s_{I}{ }^{2}$ depending on $P$ that tends to infinity when $P \rightarrow P_{c}$. The process becomes subcritical (i.e., without ignition before the adiabatic thermal explosion time associated with the initial temperature $T_{0}$ ) if $P<P_{c}$, for spot sizes smaller than the critical. For large hot spots, when $P \rightarrow \infty$, the ignition time coincides with that obtained by Kindelán and Liñan (1976) when analyzing the ignition of a semi-infinite reactive solid by a hot semiinfinite gas; $s_{I}{ }^{2}(\infty)=0.393$.

For cases where $k_{i} / k_{0}$ is large, corresponding to ignition by hot metal bodies, the analysis of Sec. V should be used to calculate the interface temperatures $T_{s}$, represented by $\phi_{s}=\left(T_{s}-T_{i}\right) E / R T_{i}{ }^{2}$, that is given in terms of the nondimensional time $\tau=\alpha_{0} t / a^{2}$ by the solution of the differential Eq. (36). For each fixed value of the ratio of heat capacities $\lambda$, an ignition time $\tau_{I}(\delta, \lambda)$, defined, following Zel'dovich (1963), as the time when the heat loss from the hot spot becomes zero, exists if $\delta$ is larger than a critical value $\delta_{c}(\lambda)$; for smaller values of $\delta$ the hot spot becomes subcritical, cooling down to the initial temperature $T_{0}$ without ignition. For large values of $\delta$ ignition takes place, without significant change in the hot-spot temperature, at a time $\tau_{l}=1 / \pi \delta$, first given by Zel'dovich (1963). This time is modified substantially when higher order terms are retained in an asymptotic expansion for large $R T_{i} / E$, as shown by Liñán and Williams (1979).

For small values of $\lambda$, corresponding to $\rho_{i} c_{i} / \rho_{0} c_{0} \sim 1$, the asymptotic analysis of Berman and Ryazantsev (1976) is reproduced, with $\delta_{c}=0.312 \lambda^{-2}$. For large values of $\lambda$, corresponding to ignition of a reacting gas by a hot solid body, $\delta_{c} \rightarrow 1$ for spherical spots and $\delta_{c} \rightarrow 0$ for wire ignition.

\section{Acknowledgment}

This research has been partially sponsored by the U.S. Army European Research Office through Grant DAERO 79-G-0007.

\section{References}

Baer A.D. and Ryan N.W. (1970) Evaluation of thermal-ignition models from hotwire ignition tests. Combustion and Flame, 15, 9.21.

Berman V.S. and Ryazantsev Iu.S. (1976) Ignition of a homogenous reacting medium by a heat source with finite heat content. Prikl. Mat. Mekhan. 40, 1065-1069.

Carslaw H.S. and Jaeger J.C. (1959) Conduction of Heat in Solids, p. 346. Oxford University Press, Oxford, England.

Crank J. (1956) The Mathematics of Diffusion, pp. 26-30. Oxford University Press, Oxford, England. 
Friedman M.H. (1969) A general thermal explosion criterion: application to initiation by imbedded wires. Combustion and Flame, 13, 567-576.

Goldshleger U.I., Pribytkov K.V., and Barzykin V.V. (1973) Ignition of a condensed explosive by a hot object of finite dimensions. Fizika Goreniya $i$ Vzryva, 9, 119123.

Kindelán M. and Liñán, A. (1976) Ignition of a reactive material by hot gases. Scientific Rept. 2, AFOSR 73-2535, INTA, Madrid, Spain, Nov. 15.

Kindelán M. and Liñan A. (1978) Gasification effects in the heterogeneous ignition of a condensed fuel by a hot gas. Acta Astronautica. 5, 1199-1211.

Letcher J.S. (1969) On boundary layers in the theory of heat conduction in solids. SIAM Rev. 11, 20-29.

Liñán A. and Williams F.A. (1979) Ignition of reactive solid exposed to a step in surface temperature. SIAM J. Appl. Math. 36, 587-603.

Merzhanov A.G. and Averson A.E. (1971) The present state of the thermal ignition theory: an invited review. Combustion and Flame 16, 89-124.

Randolph A.D. and Simpson K.D. (1973) A theoretical study of accidental initiation of encased high explosive charges by gas compression mechanisms. AFOSR Contract F44620-70-C-0088, Chemical Engineering Dept., Univ, of Arizona, Tucson.

Randolph A.D. (1975) Ignition of HE charges by a gas compression mechanism. Final Rept. AFOSR Contract F44620-70-C-0088, College of Mines, Univ. of Arizona, Tucson.

Vilyunov V.N. and Kolchin A.K. (1966) Ignition of condensed explosives by conductive heat transfer from media with poor thermal conductivity. Fizika Goreniya i Varyva, 2, 101-109.

Zel'dovich Ya.B. (1963) Theory of ignition. Dokl. Akad. Nauk., 150, 2. 